\title{
Multiagent Simulation of Physical Phenomena by Means of Aspect Programming
}

\author{
Sławomir Bieniasz, Stanisław Ciszewski, and Bartłomiej Śnieżyśki \\ AGH University of Science and Technology, Department of Computer Science, \\ Krakow, Poland \\ bieniasz@agh.edu.pl, scisz@iisg.agh.edu.pl, sniezyn@agh.edu.pl
}

\begin{abstract}
Along with the evolution of the numerical methods new software methodologies have been developed. Goal of our research is to apply Aspect Oriented Programming (AOP) in the development of multiagent simulation system. In this paper theoretical model of aspect-multiagent system is presented, its architecture and implementation is described. Results of experiments performed conclude the work. The model considered here can serve as a design tool for foundry processes, especially to design conditions for cooling of a casting leading to desired crystal structure.
\end{abstract}

\section{Introduction}

Simulation software is usually build using structural programming techniques, which resembles functions and equations found in an application domain. This leads to a clear structure of a model which is typically specialized in a narrow field of the application.

The next milestone of software engineering, object orientation paradigm, has been introduced to numerical methods last years. The advantages of this approach are well known, but on the field of simulation of natural phenomena one important drawback have to be pointed. The data centric encapsulation, which perfectly plays its role on business model field, is hard to be properly done when common state is more shared then divided.

The paper presents the next step approach to this problem i.e. composition of Agent Methodologies and Aspect Oriented Programming (AOP) [1]. In such an approach the state of the system is modelled as an environment, typically a mesh, which could be observed and changed by set of agents. The physical phenomena are represented as their societies, mutual relations and specialized roles. The separation of crosscutting concerns is used to distinguish both the physical phenomena and parts of the software.

The paper develops those ideas as follows. The second section gives the description of some related works on the field of aspect-multiagent approach. The third section presents a theoretical model of such system which is centered upon the separation of physical phenomena concept. The demonstration system Aspect MAFES is described in the forth section. The real life examples are shown 
in the fifth section. The model considered there can serve as a design tool for foundry processes, especially to design conditions for cooling of a casting leading to desired crystal structure.

\section{Related Work}

Aspect programming is used to build multiagent systems for several years. Kendal 22 presents how aspects can be used to model, design and implement agent roles, which focuses on the position and responsibilities within an overall multiagent system. Examples of roles are broker, mediator, and bureaucracy.

Garcia et al. 3] describe using aspect-oriented programming to separate concerns in multiagent systems development. Two main groups of aspects are proposed: agency aspects (such as autonomy, collaboration, and mobility), and generic aspects (e.g. exception handling, and persistance). Additionally, application of computational reflection is proposed to manipulate separately interagent level properties.

Robbes et al. 4] propose application of aspect-oriented programming in multiagent systems that are based on the Aalaadin [5] MAS model. It is done on two leveles: conceptual (where aspect is unified with the group concept) and implementation. Aspects that are proposed are connected with messaging (message building, messaging strategy, distributed messaging), agent lookup, visualizatoin and debuging, and flow control.

In this work an approach similar to the Garcia's and Robbes' is used in the numerical simulation domain.

\section{Theoretical Models}

Let us denote by $S$ a set of states of universe, by $P$ a set of observations, by $A$ a set of actions, and by $A g$ a set of agents. Classically, agent is determined as pair of methods [6]:

$$
a_{i}=\left(\text { see }_{i}, \operatorname{action}_{i}\right)
$$

where:

$$
\begin{aligned}
\text { see }_{i} & : S \rightarrow P, \\
\text { action }_{i} & : P^{\star} \rightarrow A,
\end{aligned}
$$

where $P^{\star}$ is a set of sequences of observations from $P$. Function see $_{i}$ receives information about the environment and transforms it into some internal observation. The action $_{i}$ chooses an action that should be executed according to the history of information received.

In this work an environment is treated as a deterministic state transition function:

$$
\text { env }: A \times S \rightarrow S
$$


With such assumption a consecutive application of see $_{i}$, action $_{i}$ and $e n v$ is valid, only if observations and actions that are executed are alternate. To overcome this problem the additional function:

$$
\sigma: A g \rightarrow \mathbb{N}
$$

is introduced to assign a priority to every agent. Priorities should be assigned in a way that agent actions and observations with the same priority are alternate.

To handle more sophisticated functionality, the basic structure of the agent system is extended in the following way:

- the state of the universe is modelled as a family of partial functions,

- to introduce slices, instructions used to code $s e_{i}$ and $a c t_{i}$ are considered with static data flow dependencies extended to the universe,

- finally, physical representation of aspect waving is defined.

Let $\Gamma \neq \emptyset$ be a set of entities (or attributes) in the universe. For any $\gamma \in \Gamma$ we define the set of its values $V_{\gamma}$. By a $\mathcal{V}=\bigcup_{\gamma \in \Gamma} V_{\gamma}$ the total space of values is noted. With this annotations the function:

$$
s: \Gamma \ni \gamma \rightarrow s(\gamma) \in V_{\gamma} \subset \mathcal{V}
$$

denotes the state of the universe in a given time. We assume that for all $\gamma \in \Gamma$ the set of values $V_{\gamma}$ contains a special none element. For a given state the statement $s(\gamma)=$ none means that $\gamma$ cannot be taken into account in the state $s$.

The internal structure of an agent is a pair $(\mathbb{I}, \ll)$, where $\mathbb{I}$ denotes the set of instructions, and $\ll$ is a static data flow relation. It is assumed that $\ll$ is an order, as usual. This relation is naturally extended to the entities of $\Gamma$ by instruction value dependencies.

Definition 1. The slice in $\mathbb{I}$ is a pair $A=\left(A^{\Gamma}, A^{\mathbb{I}}\right)$ where $A^{\Gamma} \subset \Gamma$ and $A^{\mathbb{I}}$ is set of instructions satisfying following conditions:

- if $i \in A^{\mathbb{I}}$ then $\forall a \in \Gamma$ such that: $a \ll i$ then $a \in A^{\Gamma}$,

- if $i \in A^{\mathbb{I}}$ and $a \in A^{\Gamma}$ then $\forall j \in \mathbb{I}$ such that: $a \ll j \ll i$ then $j \in A^{\mathbb{I}}$.

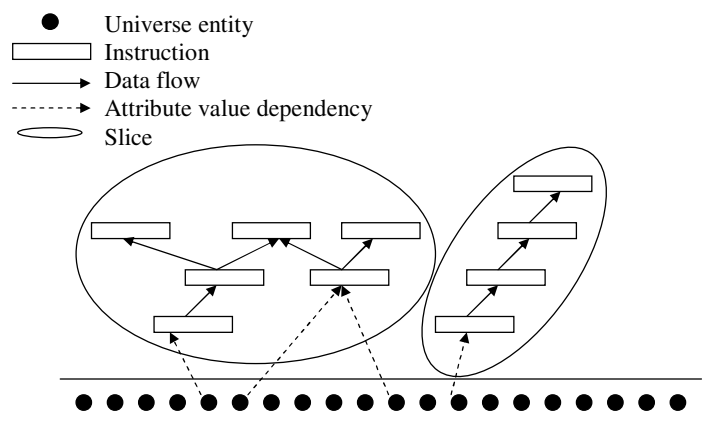

Fig. 1. Slices in the MAS 
The definition is illustrated in Fig. 1. The dots below depicts the elements of the universe. The instructions, marked as rectangles, depend on their values. The data flow relation between instructions transfer the value dependency to the further instructions. The two slices are marked by ovals.

Let us remark, that any slice $A$ could be treated as a sum of intersection with agent methods. It is denoted as: $A_{\text {see }}=A \cap$ see and $A_{\text {action }}=A \cap$ action.

Definition 2. Two slices $A$ and $B$ are independent iff $A^{\Gamma} \cap B^{\Gamma}=\emptyset$ and $A^{\mathbb{I}} \cap B^{\mathbb{I}}=\emptyset$.

Our example (Fig. 1) shows such a situation.

Definition 3. A slice $A$ is complete iff $A$ and the smallest slice which contain its complement $\left(\Gamma-A^{\Gamma}, \mathbb{I}-A^{\mathbb{I}}\right)$ are independent.

The completeness of slices $A$ should be understood, in the context of this article, as a separation of physical phenomena. Each of them could be distinguished by a set of entities and their values, which constitutes restriction of universe state to $A^{\Gamma} \subset \Gamma$.

Definition 4. A slice $A$ separates phenomenon iff there exists a complete slice $B$ such that: $A^{\Gamma}=B^{\Gamma}$ and $A^{\mathbb{I}} \subset B^{\mathbb{I}}$

Let us consider a slice $A$ and a family of $\left\{X_{i}\right\}_{i=1,2, \ldots, k}$ such that: $X_{i}=\left(X_{i}^{\Gamma}, X^{\mathbb{I}}\right)$, where: $X_{i}^{\Gamma} \subset \Gamma$ and $X_{i}^{\mathbb{I}} \subset \mathbb{I}$ for $i=1,2, \ldots, k$.

Definition 5. A slice $A$ is properly extended (weaved) by a $X_{i}$ when $A+X_{i}=$ $\left(A^{\Gamma} \cup X_{i}^{\Gamma}, A^{\mathbb{I}} \cup X_{i}^{\mathbb{I}}\right)$ is also a slice.

Definition 6. With the above notation the aspect system separates phenomena if all partial proper extensions for set $U \subset\{1,2, \ldots, k\}: A+\sum_{U} X_{i}$ separates phenomena; where $A+\sum_{U} X_{i}$ means $\left(\ldots\left(\left(A+X_{i_{1}}\right)+X_{i_{2}}\right) \ldots+X_{i_{n}}\right)$ for $i_{j} \in U$, $j \in\{1,2, \ldots, n\}, n=|U|$.

\section{System Description}

During refactoring of the MAFES system (Multi Agent Finite Environment System) 7] several aspects were identified. As a result an Aspect MAFES system was built according to a theoretical model described above.

System consists of a multiagent basic model, with which separate phenomena, control, and visualization aspects are weaved.

Multiagent basic model consist of an environment in the form of the node matrix, and a set of agents operating on these nodes. The environment is used to model a space. Every node has references to the closest neighbors. Agents observe the universe state, and affect the part of it, by changing selected entities values, which are in theirs activity range.

Aspects are used to assign tasks to agents. Task assignment is connected with adding appropriate functionality to agents to perform given task and weaving 
appropriate resources and attributes to the universe. Aspects are used to add physical phenomenons (such as motion, heat exchange, crystallization, etc.) and other activities (control, visualization, storing simulation results, etc.).

The following physical phenomenon aspects are implemented in the system: heat exchange, crystallization, and motion. Schemas of these aspects are presented in Fig. 2.

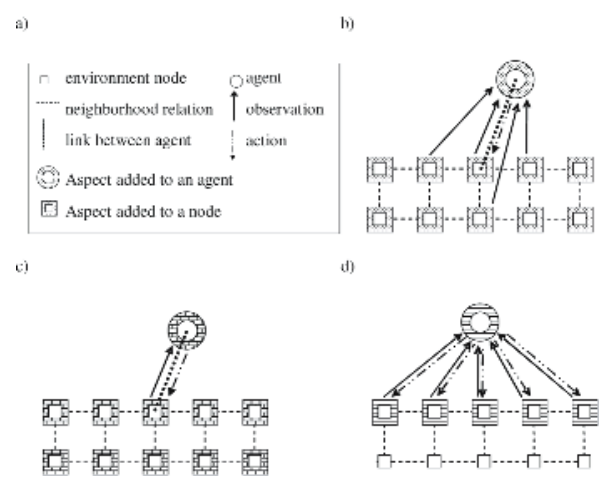

Fig. 2. Aspects of phenomena: a) legend, b) aspect of heat exchange, c) aspect of crystallization, d) aspect of motion

Aspect of heat exchange (Fig. 2-b) adds to the environment nodes the following properties: temperature, specific heat, heat conductivity, and density. To the agent activity temperature change algorithm is added. According to the theoretical model, it is build of two parts: observation that gets temperature and other parameters of the linked node and its neighbors, and action that estimates differentials of the temperature and changes the temperature of the linked node.

Aspect of crystallization (Fig. 2fc) is implemented using model of eutectic equilibrium. It adds to the environments attributes representing all necessary crystallization parameters (FS, N, R, CGN). Agent with this aspect weaved observes temperature and these parameters. Using actual and historical observations (history is necessary to recognize undercooling point), it estimates temperature change and applies it to the linked node.

Aspect of motion (Fig. 2-d) causes that agent observes and affects all moving space (nodes that represent it). Such an agent transfers properties of these nodes according to the speed of motion. This action should be done before these mentioned above. As a result, agents with this aspect weaved have higher priority.

Three more aspects are used in Aspect MAFES: control (e.g. used to control the cooling), visualization (to present the state of simulation) and result storing (to store all necessary simulation parameters). Because of the lack of space, they are not described here.

Aspect MAFES is implemented using AspectJ [8, 9] language, a simple, uniform and very practical extension of Java. This language adds several tools that provide a support to modular implementation of crosscuting concerns. 
Aspects extend an environment using introductions to nodes. Agents functionality is extended by defining cut points and advices.

\section{$5 \quad$ Experiments}

To test the Aspect MAFES system, several experiments were done. Results of two of them are presented below.

\subsection{First Experiment: Heat Exchange and Motion}

Two phenomena are considered in this experiment: heat exchange and a motion. A temperature map for a sequence of time stamps is presented in Fig. 3.(I). Thermophysical parameters for layers are presented in Table 1. They are chosen in a way that heat exchange in the upper layer is much slower then in the lower layer.

(I)
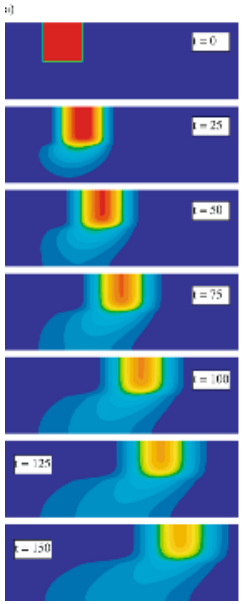

\section{")}
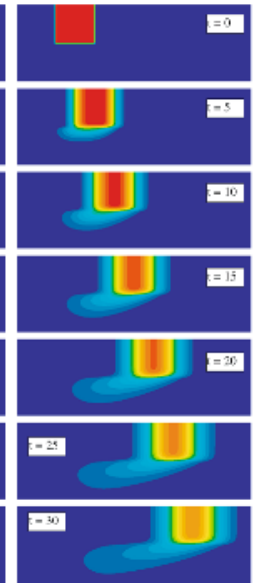

(II)
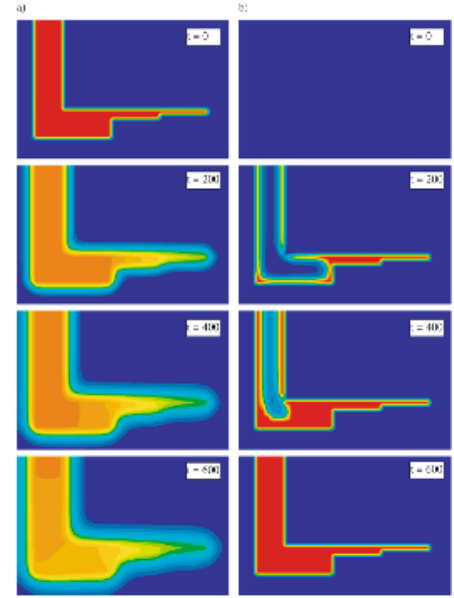

Fig. 3. (I) - temperature map for consequent time stamps in the first experiment - in the right column upper layer is moving five times faster then in the left column; (II) temperature (left column) and FS coefficient (right column) maps for consequent time stamps in the second experiment

Table 1. Termophysical parameters used in experiments

\begin{tabular}{lcccc}
\hline Parameter & \multicolumn{2}{c}{ First Experiment } & \multicolumn{2}{c}{ Second Experiment } \\
\cline { 2 - 5 } & Lower layer Upper layer & Cast & Mould \\
\hline \hline Heat conductivity $[\mathrm{W} / \mathrm{K} \mathrm{b}]$ & 20 & 5 & 210 & 1 \\
Specific heat $[\mathrm{J} /(\mathrm{kg} \mathrm{K})]$ & 300 & 1180 & 1180 & 1333 \\
Density $[\mathrm{kg} / \mathrm{m} 3]$ & 1200 & 2250 & 2550 & 1500 \\
Latent heat [J / kg] & - & - & $3.73 \mathrm{E}+5$ & - \\
Eutectic temperature $\left[{ }^{\circ} \mathrm{C}\right]$ & - & - & 610 & - \\
\hline
\end{tabular}


Two speeds of the upper layer $v_{u}$ were tested. Left column (a) is a result for the speed $v_{u}=0.002[\mathrm{~m} / \mathrm{s}]$, right (b) is for $v_{u}=0.01[\mathrm{~m} / \mathrm{s}]$. Time stamps are chosen in a way that pictures in the same row have the same positions of the upper layer.

\subsection{Second Experiment: Heat Exchange and Crystallization}

In this experiment heat exchange and crystallization in a foundry process model are considered. Cast has a stepped shape. Results of simulation are presented in Fig. 3-(II). Rows represent consecutive time stamps. In the left column (a) temperature map is presented, in the right column (b), FS coefficient, which represent the fraction of the solid phase, is shown. Termophysical parameters for mould and cast are presented in Table 1 Initially, temperature of the cast is $700\left[{ }^{\circ} \mathrm{C}\right]$, and of the mould is $20\left[{ }^{\circ} \mathrm{C}\right]$.

The shape of a cast causes a big difference in the speed of cooling down of specific regions. As a consequence, microcrystalline structure is non-uniform.

\section{Conclusions}

Presented concept of the aspect-multiagent simulation system may be applied to a wide variety of practical problems, which must take into consideration cooperation of superimposed processes occurring within the common environment.

Some experiments show usefulness of the proposed computational approach. Further development will include improvement of system versatility through incorporation of other agent and aspect types as well as adaptation to other applications.

There are several main advantages resulting from AOP application during development of the simulation system:

- there is a straight correspondence between physical phenomenon (used during analysis) and aspect (used in software development);

- code for the every physical phenomenon and other concerns (such as control, visualization, storing results) is modularized (written in one unit of compilation);

- there is an analogy between phenomenon overlapping and aspect weaving;

- building versions of the system for specific requirements is very simple (it is enough to weave appropriate aspects);

- implementation of configuration of the system is easier;

As a result, structure of the code is much simpler and easy to understand and maintain.

\section{References}

1. Kiczales, G., Lamping, J., Mendhekar, A., Maeda, C., Lopes, C., Loingtier, J.M., Irwing, J.: Aspect-oriented programming. In: Proc. of ECOOP'97, Springer Verlag (1997) 220-242

2. Kendall, E.: Aspect-oriented programming for role models. In: In Proc. of The Aspect-Oriented Programming Workshop at ECOOP'99. (1999) 
3. Garcia, A., Chavez, C., Silva, O., Silva, V., Lucena, C.: Promoting advanced separation of concerns in intra-agent and inter-agent software engineering. In: Workshop on Advanced Separation of Concerns in Object-oriented Systems (ASoC) at OOPSLA'2001, Tampa Bay, Florida, USA (2001)

4. Robbes, R., Bouraqadi, N., Stinckwich, S.: An aspect-based multi-agent system. In: Research Track of the ESUG 2004 Smalltalk Conference, Kthen (Anhalt), Germany (2004)

5. Ferber, J., Gutknecht, O.: A meta-model for the analysis and design of organizations in multi-agent systems. In: Third International Conference on Multi-Agent Systems (ICSMAS98). (1998) 128-135

6. Weiss, G., ed.: Multiagent Systems: A Modern Approach to Distributed Artificial Intelligence. The MIT Press, London (1999)

7. Bieniasz, S., Cetnarowicz, K., Nawarecki, E., Kluska-Nawarecka, S.: Agentbased simulation in finite element environment. In Binder, Z., ed.: Management and Control of Production and Logistics: a proceedings volume from the 2nd IFAC/IFIP/IEEE conference. Volume 1., Grenoble, Oxford, Pergamon (2001) 545550

8. AspectJ Team: The AspectJ documentation. (http://www.eclipse.org/aspectj/)

9. Kiczales, G., Hilsdale, E., Hugunin, J., Kersten, M., Palm, J., Griswold, W.: An overview of AspectJ. In: Proc. of the ECOOP 2001. Volume 2072 of Lecture Notes in Computer Science., Springer (2001) 